\title{
Dynamic observation-prediction for LTI systems with a time-varying delay in the input
}

\author{
V. Léchappé, E. Moulay and F. Plestan
}

\begin{abstract}
A predictive-based controller is proposed to control LTI systems in presence of time-varying delay in the input. The control method is based on the computation of an approximated (or asymptotic) prediction thanks to a dynamic system. Then, this prediction is "plugged" into any Lipschitz controller that stabilizes the delay-free system. Explicit conditions that guarantee the closed-loop stability are given thanks to a Lyapunov-Krasovskii analysis. A qualitative analysis of these conditions is performed and the results are illustrated in simulation.
\end{abstract}

Index Terms-Prediction-based control, time-varying delay, dynamic prediction, Lyapunov-Krasovskii functional

\section{INTRODUCTION}

Input delay systems are a subclass of time delay systems (TDS). The reader can refer to survey papers [1] and [2] for a general review on TDS. An input can be delayed because of a large computation time or because of latencies during communications. The latter example is particularly common for remote controlled devices such as UAVs, satellites or in Networked Control Systems (NCS).

There exist two different approaches to control input delay systems: memoryless (or memory free) and memory controllers. The advantage of memory free controllers is that they do not require the computation of an integral. See for example [3] for bounded control, [4] for adaptive control, [5] for a truncated predictor and [6] for sliding mode techniques. The drawback is that they usually cannot guarantee a good level of performance for unstable systems with large delays.

In this case, memory controllers can be designed. For systems with a single delay in the input (as those considered in this work), a memory controller is often a controller based on the computation of a prediction. It has been highlighted in [7] that state prediction is a fundamental concept for delay systems, much like state observation is for systems with incomplete state measurements. The most well-known method to control dead time systems is probably the Smith predictor. This frequency approach was introduced by Smith at the end of the 1950s in [8]. At the end of the 1970s and the beginning of the $1980 \mathrm{~s}$, the result of Smith has been extended to state-space representation and unstable systems by [9], [10] and [11].

Vincent Léchappé is with the University of South Pacific, School of Engineering and Physics, Laucala campus, Suva, Fiji. lechappe.vincent@gmail.com

Emmanuel Moulay is with Xlim, UMR CNRS 7252, Université de Poitiers emmanuel.moulay@univ-poitiers.fr

Franck Plestan is with LUNAM Université, Ecole Centrale de Nantes, IRCCyN UMR CNRS 6597, Nantes, France. franck.plestan@irccyn.ec-nantes.fr.
The inconvenient of these methods is that they usually require the discretization of an integral. For open-loop stable systems, the integral term can be computed without discretizing the integral [12]. However, for open-loop unstable systems, the integral has to be discretized in a finite number of points. This step has to be done very carefully since it can destabilize the system as pointed out in [13]. A prediction approach based on an approximated prediction computed thanks to a dynamic system has been proposed for the first time in [14] for the control of input delay systems. The advantage is that no discretization is required to compute the prediction. The idea has also been used for the observation of systems with delayed output [15], [16], [17]. Recently, some works have used the same idea of dynamic prediction for control purposes: [18] with full state knowledge, [19] and [20] considering sample and hold phenomena.

However, the works mentioned above consider a constant delay. In this article, the time-varying delay-case is treated by adding an extra term in the Lyapunov-Krasovskii functionals. The exponential stability is proven for a large class of controllers and observers. In addition, explicit conditions for stability are given and analyzed.

The paper is organized as follows. The different assumptions and the problem are stated in Section II. In Section III, the dynamic observer-predictor is presented along with a convergence proof and a qualitative analysis of the stability conditions. This result is extended to the case of various sub observers-predictors in Section IV. In Section V, simulations support previous theoretical results. Finally, some perspectives are given in Section VI.

\section{Problem StATEMEnT}

The class of systems considered in this work is the following

$$
\left\{\begin{array}{l}
\dot{x}(t)=A x(t)+B u(t-h(t)) \\
y(t)=C x(t)
\end{array}\right.
$$

where $h(t)$ is a known and time-varying delay. The objective is to design prediction-based controllers without the need of computing the exact prediction. Indeed, the discretization is delicate process that often requires a large computing power that may restrict the application range of predictive methods in practice. In addition, for a time-varying delay, future values of the delay have to be known in advance to compute the exact prediction [21], [22]. In this article, only the value of $h$ has to be known at instant $t$. The controller is designed for the delay-free system and then the prediction 
is "plugged" in it. As a consequence, throughout this paper it is assumed that

Assumption 1: There exists a time differentiable and globally Lipschitz controller $u: x \mapsto u(x)$, that guarantees the existence a function $V_{u}: \mathbb{R}^{n} \rightarrow \mathbb{R}$

$$
V_{u}(x)=x^{T} P x
$$

with $P$ a $n \times n$ symmetric matrix that satisfies the inequalities

$$
\left\{\begin{array}{l}
\underline{c}\|x\|^{2} \leq V_{u}(x) \leq \bar{c}\|x\|^{2} \\
\dot{V}_{u}(x(t)) \leq-c_{u}\|x(t)\|^{2}, \\
\left\|\frac{d V_{u}}{d x}\right\| \leq c\|x\|,
\end{array}\right.
$$

with $\underline{c}, \bar{c}, c_{u}, c>0$.

Remark 2.1: Note that the argument " $t$ " in $u(x(t))$ will be omitted for clarity. Furthermore, the notations $u(t)$ or $u(x)$ will be used indifferently when no confusion is possible.

Assumption 1 implies that the controller $u(x)$ globally exponentially stabilizes delay-free system. Note that Theorem 4.14 in [23] guarantees the existence of a Lyapunov function that verifies (3) for a general class of exponentially stable nonlinear systems. However, here a special form (but classic) of the Lyapunov function $\left(V_{u}=x^{T} P x\right)$ is assumed. Remark that differentiating $V_{u}$ along the trajectories of the delay-free system and using inequalities (3), one gets

$$
x^{T}\left[A^{T} P+P A\right] x+u^{T} B^{T} P x+x^{T} P B u \leq-c_{u}\|x\|^{2} .
$$

In addition, since $u: x \mapsto u(x)$ is globally Lipschitz, there exists $l_{u}>0$ such that

$$
\|u(x)\| \leq l_{u}\|x\|
$$

for all $x \in \mathbb{R}^{n}$. It is also assumed that an estimation $\hat{x}$ of the state $x$ can be computed thanks to an observer:

$$
\dot{\hat{x}}(t)=A \hat{x}(t)+B u(t-h(t))+g(C \hat{x}(t)-y(t))
$$

where the correction term $g$ is such that

Assumption 2: There exists a globally Lipschitz function $g$ that guarantees the existence of a function $V_{g}: \mathbb{R}^{n} \rightarrow \mathbb{R}$

$$
V_{g}(e)=e^{T} Q e
$$

with $e=\hat{x}-x$ and $Q$ a $n \times n$ symmetric matrix that satisfies the inequalities

$$
\left\{\begin{array}{l}
\frac{m}{\dot{V}_{g}}\|e\|^{2} \leq V_{g}(e) \leq \bar{m}\|e\|^{2}, \\
\left\|\frac{d V_{g}}{d e}\right\| \leq m\|e\|,
\end{array}\right.
$$

with $\underline{m}, \bar{m}, m_{u}, m>0$.

Remark 2.2: By a slight abuse of notation, $g(\mathrm{Ce})$ will be sometimes denoted $g(e)$ to underline that it depends on the observation error $e$.

Assumption 2 implies that the function $g$ globally exponentially stabilizes the observation error

$$
\dot{e}(t)=A e(t)+g(C e(t)) .
$$

Similarly to (4), one gets

$$
e^{T}\left[A^{T} Q+Q A\right] e+g^{T}(C e) Q e+e^{T} Q g(C e) \leq-m_{g}\|e\|^{2} .
$$

In addition, since $g$ is globally Lipschitz, there exists $l_{g}>0$ such that

$$
\|g(C e)\| \leq l_{g}\|C e\|
$$

for all $e \in \mathbb{R}^{n}$. Some assumptions are also made to characterize the time-varying delay $h(t)$ :

Assumption 3: The time-varying delay $h(t)$ is upper and lower bounded by strictly positive constants.

Assumption 4: The delay is differentiable and its dynamics is bounded.

In the next section, the dynamic observation-prediction method is presented.

\section{DYNAMIC OBSERVATION AND PREDICTION METHOD}

The observation and the prediction are computed by a single dynamic system

$$
\dot{z}(t)=A z(t)+B u(t)+g(C z(t-h(t))-y(t)])
$$

with $g$ a correction term verifying Assumption 2 .

Remark 3.1: Note that to compute $z(t)$, the initial condition $z(t)=\phi_{z}(t)$ for $t \in\left[-h_{\max }, 0\right]$ with $\phi_{z}$ a continuous function, is required. The prediction $z$ will be continuous in $t=h(t)$. Also remark that the variable $z(t)$ has to be stored on $\left[t-h_{\max }, t\right]$ which imposes constraints on practical implementations.

Then, a predictive feedback $u(z)$ with $u$ that verifies Assumption 1 can be applied to stabilize (1). Notice that the non-delayed input $u(t)$ enters system (12) and that the correction term involves the delayed estimation state $z(t-h(t))$, that is why $z(t)$ is an approximation of $x(t+h(t))$.

In order to state the next result, the prediction error is denoted by

$$
e_{p}(t)=z(t-h(t))-x(t) .
$$

It is now possible to introduce the following theorem.

Theorem 1: Consider system (1), where $h(t)$ is known and complies with Assumptions 3 and 4, and assume that there exist a controller $u$ and the correction term $g$ satisfying Assumptions 1 and 2 respectively. Suppose that system (1) is controlled by $u(z)$ with $z$ computed by observer-predictor (12) and define

$\Upsilon(t)=\|x(t)\|^{2}+\sup _{s \in\left[t-h_{\max }, t\right]}\|z(s)\|^{2}+\sup _{s \in\left[t-h_{\max }, t\right]}\left\|\dot{e}_{p}(s)\right\|^{2}$.

Then, there exist $\varsigma, \varrho, h^{*}, \delta^{*}>0$ such that, provided

$$
h(t)<h^{*} \text { and }|\dot{h}(t)|<\delta^{*},
$$

one has

$$
\Upsilon(t) \leq \varsigma \Upsilon(0) e^{-\varrho t} \quad \forall t \geq 0
$$

therefore $\lim _{t \rightarrow+\infty}\|x(t)\|=0$.

Proof: Note that the delay $h(t)$ (respectively $\dot{h}(t)$ ) will be denoted $h$ (respectively $\dot{h}$ ) in the proof to facilitate the reading. First, denoting $e_{p}(t)=z(t-h(t))-x(t)$, equation (12) becomes

$$
\dot{z}(t)=A z(t)+B u(t)+g\left(C e_{p}(t)\right) .
$$


The dynamics of $e_{p}(t)$ reads as

$$
\begin{aligned}
\dot{e}_{p}(t)= & A e_{p}(t)+g\left(C e_{p}(t-h)\right) \\
& -\dot{h}\left[A z(t-h)+B u(t-h)+g\left(C e_{p}(t-h)\right)\right]
\end{aligned}
$$

and can be rewritten as follows

$$
\begin{aligned}
\dot{e}_{p}(t)= & A e_{p}(t)+g\left(C e_{p}(t)\right)-\int_{C e_{p}(t-h)}^{C e_{p}(t)} \frac{d g}{d s}(s) d s \\
& -\dot{h}\left[A z(t-h)+B u(t-h)+g\left(C e_{p}(t-h)\right)\right] .
\end{aligned}
$$

Since the delay is bounded from Assumption 3, there exist $h_{\min }, h_{\max }>0$ such that

$$
h_{\min } \leq h(t) \leq h_{\max }
$$

The following Lyapunov-Krasovskii functional candidate is proposed

$$
V(t)=\gamma V_{1}(t)+V_{2}(t)+V_{3}(t)+V_{4}(t)
$$

where

$$
V_{1}(t)=z^{T}(t) P z(t)
$$

with $P$ defined in (2),

$$
V_{2}(t)=e_{p}^{T}(t) Q e_{p}(t),
$$

with $Q$ defined in (7),

$$
V_{3}(t)=\int_{t-h_{\max }}^{t}\left(h_{\max }+s-t\right)\left\|\dot{e}_{p}(s)\right\|^{2} d s
$$

$V_{4}(t)=\int_{t-h}^{t}\left(h_{\max }+h_{\min }+s-t\right)\left(\|z(s)\|^{2}+\left\|e_{p}(s)\right\|^{2}\right) d s$

and $\gamma>0$. Since $g$ is globally Lipschitz and from (4), the time derivatives of $V_{1}$ satisfies

$$
\dot{V}_{1}(t) \leq-c_{u}\|z(t)\|^{2}+R\|z(t)\|\left\|e_{p}(t)\right\|
$$

with $R=2 l_{g}\|P\|\|C\|$. Moreover, by substitution one gets

$$
\left\|\int_{C e_{p}(t-h(t))}^{C e_{p}(t)} \frac{d g}{d s}(s) d s\right\| \leq l_{g}\|C\| \int_{t-h(t)}^{t}\left\|\dot{e}_{p}(s)\right\| d s,
$$

so using (10), it follows that

$$
\begin{aligned}
\dot{V}_{2}(t) \leq & -m_{g}\left\|e_{p}(t)\right\|^{2}+R^{\prime}\left\|e_{p}(t)\right\|\|m(t)\| \\
& +|\dot{h}| M\left\|e_{p}(t)\right\|\|z(t-h)\| \\
& +|\dot{h}| R^{\prime}\left\|e_{p}(t)\right\|\left\|e_{p}(t-h)\right\|
\end{aligned}
$$

with $R^{\prime}=2 l_{g}\|Q\|\|C\|, M=2\|P\|\left(\|A\|+l_{u}\|B\|\right)$ and $\|m(t)\|=\int_{t-h}^{t}\left\|\dot{e}_{p}(s)\right\| d s$. Furthermore, using Leibniz differentiation rule, one gets

$$
\dot{V}_{3}(t)=h_{\max }\left\|\dot{e}_{p}(t)\right\|^{2}-\int_{t-h_{\max }}^{t}\left\|\dot{e}_{p}(s)\right\|^{2} d s
$$

so, reminding that

$$
\begin{aligned}
-\int_{t-h_{\max }}^{t}\left\|\dot{e}_{p}(s)\right\|^{2} d s & \leq-\int_{t-h(t)}^{t}\left\|\dot{e}_{p}(s)\right\|^{2} d s \\
& \leq-\frac{1}{h_{\max }}\left(\int_{t-h(t)}^{t}\left\|\dot{e}_{p}(s)\right\| d s\right)^{2},
\end{aligned}
$$

it follows that

$$
\begin{aligned}
\dot{V}_{3}(t) \leq & h_{\max }\left\|\dot{e}_{p}(t)\right\|^{2}-\frac{1}{2 h_{\max }}\|m(t)\|^{2} \\
& -\frac{1}{2} \int_{t-h_{\max }}^{t}\left\|\dot{e}_{p}(s)\right\|^{2} d s .
\end{aligned}
$$

In addition, since $u$ and $g$ are globally Lipschitz and using (26) and Hölder's inequality, it can be deduced from (18) that

$$
\begin{aligned}
\left\|\dot{e}_{p}\right\|^{2} \leq & c_{1}\left\|e_{p}\right\|^{2}+c_{2}\|m\|^{2}+c_{3}|\dot{h}|^{2}\|z(t-h)\|^{2} \\
& +c_{2}|\dot{h}|^{2}\left\|e_{p}(t-h)\right\|^{2}
\end{aligned}
$$

with $c_{1}=4\left(\|A\|+l_{g}\|C\|\right)^{2}, c_{2}=4 l_{g}^{2}\|C\|^{2}, c_{3}=4(\|A\|+$ $\left.l_{u}\|B\|\right)^{2}$ so

$$
\begin{aligned}
\dot{V}_{3}(t) \leq & h_{\max }\left[c_{1}\left\|e_{p}\right\|^{2}+c_{2}\|m\|^{2}+c_{3}|\dot{h}|^{2}\|z(t-h)\|^{2}\right. \\
& \left.+c_{2}|\dot{h}|^{2}\left\|e_{p}(t-h)\right\|^{2}\right]-\frac{1}{2 h_{\max }}\|m(t)\|^{2} \\
& -\frac{1}{2} \int_{t-h_{\max }}^{t}\left\|\dot{e}_{p}(s)\right\|^{2} d s .
\end{aligned}
$$

Finally,

$$
\begin{aligned}
\dot{V}_{4}(t) \leq & \left(h_{\max }+h_{\min }\right)\left(\|z(t)\|^{2}+\left\|e_{p}(t)\right\|^{2}\right) \\
& -h_{\min }(1-|\dot{h}|)\left(\|z(t-h)\|^{2}+\left\|e_{p}(t-h)\right\|^{2}\right) \\
& -\int_{t-h}^{t}\left(\|z(s)\|^{2}+\|e(s)\|^{2}\right) d s .
\end{aligned}
$$

Thus, using Young's inequality, the "completing the square" method and Assumptions 1 and 2, the following inequality is obtained

$$
\begin{aligned}
\dot{V}+\varepsilon V \leq & -\left[\frac{\gamma c_{u}}{2}-2 h_{\max }-\gamma \varepsilon \bar{c}\right]\|z(t)\|^{2} \\
& -\left[\frac{1}{2 h_{\max }}-\frac{R^{\prime 2}}{2 m_{g}}-h_{\max } c_{2}\right]\|m(t)\|^{2} \\
& -\left[\frac{m_{g}}{2}-\frac{\gamma R^{2}}{2 c_{u}}-h_{\max }\left(2+c_{1}\right)\right. \\
& \left.-|\dot{h}|\left(M / 2+R^{\prime} / 2\right)-\varepsilon \bar{m}\right]\left\|e_{p}(t)\right\|^{2} \\
& -\left[h_{\min }-|\dot{h}|\left(h_{\min }+|\dot{h}| h_{\max } c_{3}+M / 2\right)\right]\|z(t-h)\|^{2} \\
& -\left[h_{\min }-|\dot{h}|\left(h_{\min }+|\dot{h}| h_{\max } c_{2}+R^{\prime} / 2\right)\right]\left\|e_{p}(t-h)\right\|^{2} \\
& -\left[\frac{1}{2}-\varepsilon h_{\max }\right] \int_{t-h_{\max }}^{t}\left\|\dot{e}_{p}(s)\right\|^{2} d s \\
& -\left[1-2 \varepsilon h_{\max }\right] \int_{t-h}\left(\|z(s)\|^{2}+\left\|e_{p}(s)\right\|^{2}\right) d s .
\end{aligned}
$$

with $\varepsilon>0$. To make $\dot{V}(t)+\varepsilon V(t) \leq 0$, it is sufficient to have the coefficients pre-multiplying the quadratic terms 
negative. It leads to the following conditions

$$
\left\{\begin{array}{l}
\frac{\gamma c_{u}}{2}-2 h_{\max }-\gamma \varepsilon \bar{c}>0, \\
\frac{1}{2 h_{\max }}-\frac{R^{\prime 2}}{2 m_{g}}-h_{\max } c_{2}>0, \\
{\left[\frac{m_{g}}{2}-\frac{\gamma R^{2}}{2 c_{u}}-h_{\max }\left(2+c_{1}\right)\right.} \\
\left.-|\dot{h}|\left(M / 2+R^{\prime} / 2\right)-\varepsilon \bar{m}\right]>0, \\
h_{\min }-|\dot{h}|\left(h_{\min }+|\dot{h}| h_{\max } c_{3}+M / 2\right)>0, \\
h_{\min }-|\dot{h}|\left(h_{\min }+|\dot{h}| h_{\max } c_{2}+R^{\prime} / 2\right)>0, \\
\frac{1}{2}-\varepsilon h_{\max }>0, \\
1-2 \varepsilon h_{\max }>0 .
\end{array}\right.
$$

First, (35), (40) and (41) can be reformulated as follows: $h_{\max }<h_{1}$ and $h_{\max }<h_{2}$ and $h_{\max }<h_{3}$ with

$$
h_{1}=\frac{\gamma}{2}\left[\frac{c_{u}}{2}-\varepsilon \bar{c}\right] \quad \text { and } \quad h_{2}=\frac{1}{2 \varepsilon} .
$$

Besides, (36) can be rewritten in the following form

$$
h_{\max }<\frac{\sqrt{\left(\frac{R^{\prime 2}}{2 m_{g}}\right)^{2}+2 c_{2}}-\left(\frac{R^{\prime 2}}{2 m_{g}}\right)}{2 c_{2}}<h_{3}
$$

with

$$
h_{3}=\frac{1}{\sqrt{2 c_{2}}} .
$$

Conditions (38)-(39) depend simultaneously on $h_{\max }$ and $|\dot{h}|$. From Assumption 4, there exists $\delta>0$ such that $|\dot{h}(t)|<$ $\delta$. Thus, rearranging the terms gives $h_{\max }<h_{4}$ and $h_{\max }<$ $h_{5}$ with

$$
h_{4}=\frac{1}{c_{3} \delta^{2}}\left[h_{m i n}-\delta\left(h_{m i n}+M / 2\right)\right]
$$

and

$$
h_{5}=\frac{1}{c_{2} \delta^{2}}\left[h_{\min }-\delta\left(h_{\min }+R^{\prime} / 2\right)\right] .
$$

Similarly condition (37) depends simultaneously on $h_{\max }$ and $\delta$ and can be reformulated as follows $h_{\max }<h_{6}$ with

$$
h_{6}=\frac{1}{2+c_{1}}\left(\frac{m_{g}}{2}-\frac{\gamma R^{2}}{2 c_{u}}-\delta\left(M / 2+R^{\prime} / 2\right)-\varepsilon \bar{m}\right) .
$$

In order to have $h_{4}, h_{5}$ and $h_{6}$ positive, $\delta$ has to comply with the condition below

$$
\delta<\delta^{*}=\min \left(\delta_{1}, \delta_{2}, \delta_{3}\right)
$$

with

$$
\delta_{1}=\frac{h_{\min }}{h_{\min }+M / 2}, \delta_{2}=\frac{h_{\min }}{h_{\min }+R^{\prime} / 2},
$$

and

$$
\delta_{3}=\frac{2}{\left(M+R^{\prime}\right)}\left(\frac{m_{g}}{2}-\frac{\gamma R^{2}}{2 c_{u}}-\varepsilon \bar{m}\right) .
$$

Choosing $\varepsilon$ and $\gamma$ sufficiently small guarantees that $h_{1}$ and $\delta_{3}$ are positive. In that case, taking

$$
h(t)<h_{\max }<h^{*}=\min \left(h_{1}, h_{2}, h_{3}, h_{4}, h_{5}, h_{6}\right)
$$

and $|\dot{h}(t)|<\delta<\delta^{*}$ ensures that

$$
\dot{V}(t) \leq-\varepsilon V(t) .
$$

Finally, equation (15) can be deduced from (52) by lengthy but straightforward computations that are omitted here.

Remark 3.2: From (15), it can be said that the closedloop system is exponentially stable in terms of the norm $\Upsilon$ and that the convergence of $\|x\|$ to zero is exponential.

This theorem shows that a predictive feedback computed from observer-predictor (12) can stabilize system (1) provided that the delay and its variation are sufficiently small. The Lyapunov analysis that has been done to prove the stability allows to find explicit bounds for $h$ and $\dot{h}$. A qualitative analysis of these bounds $h^{*}$ and $\delta^{*}$ is given below. It is reminded that $l_{u}$ (respectively $l_{g}$ ) is the Lipschitz constant associated to the function $u$ (respectively $g$ ) and is defined in (5) (respectively (11)).

- For a small value of $\varepsilon$, then $h_{1}, h_{2}$ and $h_{6}$ increase which means that a slow convergence rate of the closedloop system allows larger value of the delay.

- Similarly, for a small value of $\varepsilon$, then $\delta_{3}$ increases which means that the delay dynamics can be faster for a slow convergence rate of the close-loop system.

- For a large value of $l_{g}$ (that appears through $c_{1}$ and $\left.c_{2}\right)$, then $h_{3}, h_{5}$ and $h_{6}$ decrease which means that the admissible delay is smaller for a fast observer.

- For a large value of $l_{u}$ (that appears through $c_{3}$ ), then $h_{4}$ decreases which means that the admissible delay is smaller for a fast controller.

- For a large value of $\delta$, then $\delta_{1}$ and $\delta_{2}$ increase which means that the delay dynamics can be faster for a small delay interval.

- For a small value of $\delta$, then $h_{4}, h_{5}$ and $h_{6}$ increase which means that a slow-varying delay can have a larger amplitude.

Simulations show that for stable systems ( $A$ Hurwitz), the real values $h^{*}$ and $\delta^{*}$ can be large. On the contrary, for unstable systems, these bounds become very small. However, the use of sequential sub observers-predictors is a solution to relax this constraint. This method is presented in the next section.

\section{SUB OBSERVERS-PREDICTORS}

The idea is similar to the one presented in [14] and [18]. However, it is extended to the time-varying delay case. In addition, the exponential stability (only asymptotic stability was proved in [18]) is proved for a larger class of observers and controllers. The technique is to design various cascaded observers-predictors; Each observer-predictor will predict the state for a fraction of the delay denoted $\bar{h}$. This time-varying prediction horizon is equal to

$$
\bar{h}(t)=\frac{h(t)}{r}, \quad r \in \mathbb{N}^{*} .
$$


The equations of the cascaded sub observers-predictors are given below

$$
\left\{\begin{array}{ccc}
\dot{z}_{1}(t)= & A z_{1}(t)+B u(t-(r-1) \bar{h}(t)) \\
& +g_{1}\left(\left[C z_{1}(t-\bar{h}(t))-y(t)\right]\right) \\
\vdots & & \\
\dot{z}_{i}(t)= & A z_{i}(t)+B u(t-(r-i) \bar{h}(t)) \\
& & +g_{i}\left(C\left[z_{i}(t-\bar{h}(t))-z_{i-1}(t)\right]\right) \\
\vdots & & \\
\dot{z}_{r}(t)= & A z_{r}(t)+B u(t) \\
& +g_{r}\left(C\left[z_{r}(t-\bar{h}(t))-z_{r-1}(t)\right]\right)
\end{array}\right.
$$

where $z_{i} \in \mathbb{R}^{n}, i=1, \ldots, r$. A predictive output feedback $u\left(z_{r}\right)$ that verifies Assumption 1 can be applied to stabilize system (1).

In order to the state the next theorem, we define the sub prediction errors

$$
e_{p_{1}}=z_{1}(t-\bar{h}(t))-x(t)
$$

and

$$
e_{p_{i}}(t)=z_{i}(t-\bar{h}(t))-z_{i-1}(t)
$$

for all $i=2, \ldots, r$. The advantage of the sequential structure is to relax the condition on the maximum delay value that is mentioned in Theorem 1 .

Theorem 2: Consider system (1), where $h(t)$ is known and complies with Assumptions 3 and 4, and assume that there exist a controller $u$ and gains $g_{i}$ satisfying Assumptions 1 and 2 respectively. Suppose that system (1) is controlled by $u\left(z_{r}\right)$ with $z_{r}$ computed by sequential observers-predictors (54). Then, there exist $r^{*} \in \mathbb{N}^{*}$ and $\delta^{*}>0$ such that, provided $r>r^{*}$ and $|\dot{h}|<\delta^{*},\|x(t)\|$ exponentially converges to zero.

The proof, omitted for space limitations, consists of a recursive analysis combined with similar computation as in Theorem 1.

Remark 4.1: In previous theorem, the exponential stability in the sense of the norm $\Upsilon$ was shown. Here, only the exponential convergence of $\|x(t)\|$ is proven because of the induction proof.

Theorem 2 shows that the condition on the delay size $h^{*}$ in Theorem 1 can be overcame by the use of sub observerspredictors. There is still a condition on the delay rate but this condition cannot be removed without any knowledge of the future values of $h$. The minimum number of sub observerpredictor has to be chosen such that $\bar{h}(t)<h^{*}$ where $h^{*}$ is defined in Theorem 1.

The result of Theorem 2 (and 1) holds for any given controller $u$ and a given correction term $g$. In practice, choosing a "slower" controller and a "slower" correction term allows to reduce the number of sub observers-predictors.

Above results are illustrated by simulation in the next section.

\section{Simulation}

An unstable second order system is used to illustrate above results

$$
\dot{x}(t)=A x(t)+B u(t-h(t))
$$

where $A=\left[\begin{array}{cc}0 & 1 \\ 0.1 & 0.1\end{array}\right]$ and $B=\left[\begin{array}{l}0 \\ 1\end{array}\right]$.

Remark 5.1: Since system (57) is unstable, memoryless predictive technique involving saturated input [3] can not be applied to stabilize this system.

The controller

$$
u(t)=K z(t)
$$

where the gain $K=[-2.1,-3.1]$ is tuned in order to place the eigenvalues of the delay-free system to -1 and -2 . A Luenberger observer has been chosen to observe and predict the state of the system so

$$
g(C e)=L C e
$$

with

$$
L=[-1.6,-0.76]^{T}
$$

The eigenvalues of $A+L C$ are -0.5 and -1 . Unlike for a simple observer, the gain $L$ can not be chosen arbitrarily large to impose a faster dynamics of the observer-predictor than of the close-loop system. Remark that controller (58) and the correction term $L C e$ comply with Assumptions 1 and 2 respectively. Different delays will be introduced and the results 1, 2 or 3 sub observers-predictors will be compared.

First, the performances of the method are checked with constant delays. On Figure 1, the effect on stability of an increasing constant delay $h$ is illustrated. Note that the delays applied in simulation have the same order of magnitude as the dynamics of the closed loop system. It can be observed on Figure 1a for $h=0.5 \mathrm{~s}$ that the system can be stabilized for 1,2 or 3 sub observers-predictors. However, when the delay is increased to $h=1 \mathrm{~s}$ on Figure 1b, the single observerpredictor does not guarantee the stability anymore. This is in accordance with Theorem 1 that states that there exists a maximum bound on the delay to preserve the stability. Finally, increasing again the delay to $h=1.7$ s makes the 2 sub observers-predictors become unstable. However, it can be seen that the stability is maintained using 3 sub observerspredictors. This is illustrates Theorem 2 that states that there exists a minimum number of sub observers-predictors to guarantee stability.

On Figure 2, a time-varying delay affects the input of system (57). One can see that the system is stabilized using 2 sub observers-predictors.

\section{CONCLUSION}

It has been shown that a single dynamic system can estimate and predict (approximately) the future state of the system for a sufficiently slow time-varying delay. The advantage of this method is that it does not require an integral discretization. Using sequential observers-predictors allows to stabilize the system for an arbitrarily long delay provided it is sufficiently slow-varying. The exponential stability in terms of a particular norm is proven and explicit bounds are provided. The extension to nonlinear systems is considered for future developments. 


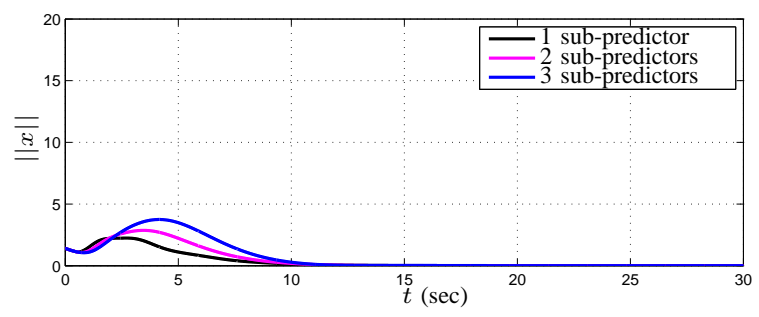

(a) $h=0.5 \mathrm{~s}$

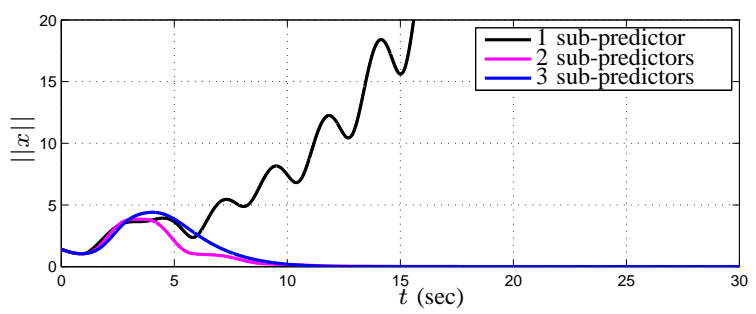

(b) $h=1 \mathrm{~s}$

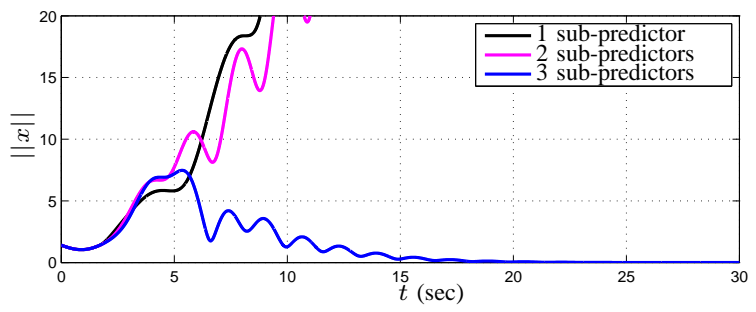

(c) $h=1.7 \mathrm{~s}$

Fig. 1: Influence of the number of sub-predictors with constant delays

\section{REFERENCES}

[1] J.-P. Richard, "Time-delay systems: an overview of some recent advances and open problems," Automatica, vol. 39, no. 10, pp. 16671694, 2003.

[2] K. Gu and S.-I. Niculescu, "Survey on recent results in the stability and control of time-delay systems," Journal of dynamic systems, measurement, and control, vol. 125, no. 2, pp. 158-165, 2003.

[3] F. Mazenc, S. Mondié, and S.-I. Niculescu, "Global asymptotic stabilization for chains of integrators with a delay in the input," IEEE Trans. Autom. Control, vol. 48, no. 1, pp. 57-63, 2003.

[4] H.-L. Choi and J.-T. Lim, "Stabilization of a chain of integrators with an unknown delay in the input by adaptive output feedback," IEEE Trans. Autom. Control, vol. 51, no. 8, pp. 1359-1363, 2006.

[5] S. Y. Yoon and Z. Lin, "Truncated predictor feedback control for exponentially unstable linear systems with time-varying input delay," Systems and Control Letters, vol. 62, no. 10, pp. 837-844, 2013.

[6] J.-P. Richard, F. Gouaisbaut, and W. Perruquetti, "Sliding mode control in the presence of delay," Kybernetica, vol. 37, no. 3, pp. 277-294, 2001.

[7] L. Mirkin and N. Raskin, "Every stabilizing dead-time controller has an observer-predictor-based structure," Automatica, vol. 39, no. 10, pp. 1747-1754, 2003.

[8] O. J. M. Smith, "Closer control of loops with dead time," Chemical Engineering Progress, vol. 53, no. 5, pp. 217-219, 1957.

[9] A. W. Olbrot, "Stabilizability, detectability, and spectrum assignment for linear autonomous systems with general time delays," IEEE Trans. Autom. Control, vol. 23, no. 5, pp. 887-890, 1978.

[10] W. Kwon and A. Pearson, "Feedback stabilization of linear systems with delayed control," IEEE Trans. Autom. Control, vol. 25, no. 2, pp. 266-269, 1980.

[11] Z. Artstein, "Linear systems with delayed controls: A reduction," IEEE Trans. Autom. Control, vol. 27, no. 4, pp. 869-879, 1982.
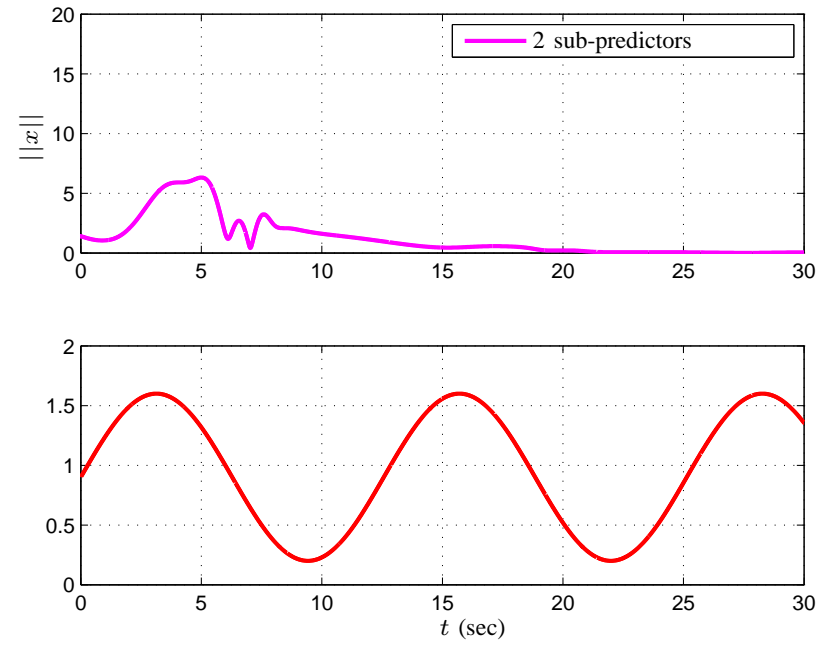

Fig. 2: Time-varying delay $h=0.9+0.7 \sin (0.5 t) \mathrm{s}$

[12] K. Watanabe and M. Ito, "A process-model control for linear systems with delay," IEEE Trans. Autom. Control, vol. 26, no. 6, pp. 12611269, 1981.

[13] V. Van Assche, M. Dambrine, J.-F. Lafay, and J.-P. Richard, "Some problems arising in the implementation of distributed-delay control laws," in Conference on Decision and Control, Phoenix, USA, 1999.

[14] G. Besançon, D. Georges, and Z. Benayache, "Asymptotic state prediction for continuous-time systems with delayed input and application to control," in European Control Conference, Kos,Greece, 2007, pp. $1786-1791$.

[15] A. Germani, C. Manes, and P. Pepe, "A new approach to state observation of nonlinear systems with delayed output," IEEE Trans. Autom. Control, vol. 47, no. 1, pp. 96-101, 2002.

[16] N. Kazantzis and R. A. Wright, "Nonlinear observer design in the presence of delayed output measurements," Systems \& Control Letters, vol. 54, no. 9, pp. 877-886, 2005.

[17] T. Ahmed-Ali, E. Cherrier, and F. Lamnabhi-Lagarrigue, "Cascade high gain predictors for a class of nonlinear systems," IEEE Trans. Autom. Control, vol. 57, no. 1, pp. 221-226, 2012.

[18] M. Najafi, S. Hosseinnia, F. Sheikholeslam, and M. Karimadini, "Closed-loop control of dead time systems via sequential subpredictors," International Journal of Control, vol. 86, no. 4, pp. 599609, 2013.

[19] T. Ahmed-Ali, I. Karafyllis, M. Krstic, and F. Lamnabhi-Lagarrigue, "Robust stabilization of nonlinear globally lipschitz delay systems," in Recent Results on Nonlinear Delay Control Systems, ser. Advances in Delays and Dynamics, M. Malisoff, P. Pepe, F. Mazenc, and I. Karafyllis, Eds. Springer International Publishing, 2016, vol. 4, pp. 43-60.

[20] I. Karafyllis, M. Krstic, T. Ahmed-Ali, and F. Lamnabhi-Lagarrigue, "Global stabilisation of nonlinear delay systems with a compact absorbing set," International Journal of Control, vol. 87, no. 5, pp. 1010-1027, 2014.

[21] M. Krstic, "Lyapunov tools for predictor feedbacks for delay systems: Inverse optimality and robustness to delay mismatch," in American Control Conference, Seattle, USA, 2008.

[22] M. T. Nihtila, "Adaptive control of a continuous-time system with time-varying input delay," Systems \& Control Letters, vol. 12, no. 4, pp. 357-364, 1989.

[23] H. K. Khalil, Nonlinear Systems, 3rd ed. Prentice Hall, 2002. 